\title{
A model integrating patch dynamics, competing species and the intermediate disturbance hypothesis
}

\author{
Belinda Barnes ${ }^{a, *}$, H.S. Sidhu ${ }^{b}$, S.H. Roxburgh ${ }^{a}$ \\ a CRC for Greenhouse Accounting, Research School of Biological Sciences, Australian National University, Canberra, ACT 0200, Australia \\ b School of Physical, Environmental and Mathematical Sciences, UNSW at ADFA, Canberra, ACT 2600, Australia
}

\section{A R T I C L E I N F O}

Article history:

Received 9 February 2005

Received in revised form 27 September 2005

Accepted 21 October 2005

Published on line 27 December 2005

Keywords:

Self-thinning

Competing species

Intermediate disturbance

hypothesis

Patch dynamics

Population dynamics

Dynamical modeling

\begin{abstract}
A B S T R A C T
Barnes and Roderick [Barnes, B., Roderick, M., 2004. An ecological framework linking scales across space and time based on self-thinning. Theor. Population Biol. 66, 113-118] developed a mathematical model of patch dynamics, based on a time-dependent self-thinning mechanism. In this paper, we consider the ramifications of their modelling approach, in terms of its agreement with the concepts of competing species and the intermediate disturbance hypothesis. When considering more than one species we show that a form of the competing species model emerges as a natural consequence of the mathematics, with our model differing from traditional competition models in its derivation and nonlinear, time-dependent per capita growth rate. Further, we show that when disturbance is included in the formulation, the general predictions are in agreement with the intermediate disturbance hypothesis.
\end{abstract}

(c) 2005 Elsevier B.V. All rights reserved.

\section{Introduction}

Barnes and Roderick (2004) proposed a simple dynamical model, based on a self-thinning mechanism, to describe a patch of vegetation in terms of changing mass (and volume) with time, where a patch referred to a group of individuals or plants. In this paper, our purpose is to show that by adopting this mathematical formulation, the concept of competing species is a natural consequence of the mathematics and, with the inclusion of system disturbance, the model encapsulates the intermediate disturbance hypothesis, also as a consequence of the formulation. While many other models encapsulate these phenomena (for example, Caswell and Etter, 1999; Roxburgh et al., 2004; Yamamoto and Hatta, 2004, or for a review, see Shea et al., 2004), the model con- sidered here differs significantly by offering a more general approach.

The model considered here incorporates self-thinning as a within-patch mechanism, well known to link the dynamics of individual plants and trees with the dynamics of the patch as a whole. Commonly, allometrics are applied to establish numbers of individuals and their average mass within the patch, and there exists a plethora of literature concerned with this approach (for example, Niklas, 1994; Niklas and Enquist, 2002). In this model, we apply a crowding factor (as developed in Barnes and Roderick, 2004 and similar to Clark, 1993), which can be linked to the allometric approach, but which allows for a dynamic relationship between the number and average mass of the individuals, and provides stability to the system.

\footnotetext{
* Corresponding author. Tel.: +61 2 61250172; fax: +61 261255095.

E-mail address: Belinda.Barnes@anu.edu.au (B. Barnes). 
A form of the competition model emerges as a direct consequence of this modeling approach. Although competition models are well known and extensively analysed (see, for example, Chesson, 1994 or Chesson, 2000a), our model differs through the inclusion of self-thinning and the emergence of a nonlinear per-capita growth function (see also Chesson, 2000b).

Diversity can arise in many ways, resulting from a variety of nonlinear mechanisms. The intermediate disturbance hypothesis is considered an explanation for the long-term diversity within ecosystems undergoing disturbance regimes, across a number of time scales. Disturbance in this sense alters the balance achieved through species competition, facilitating the relative advantage of inferior species and preventing eventual competitive exclusion; for example, it provides a mechanism for space availability, which may then be utilized by young individuals. We consider here temporal disturbance and the dynamics underlying a within-patch (sensu, Roxburgh et al., 2004) intermediate disturbance hypothesis. We do not purport to provide a general explanation for the hypothesis, but rather to illustrate that the mathematical results ensuing from the Barnes-Roderick approach are in agreement with the arguments of the hypothesis.

We begin in Section 2 with definitions, a description of the general model and the relevant assumptions. In Section 3 we establish how this formulation models competing species and is in agreement with the intermediate disturbance hypothesis. Finally, we discuss the ramifications of our results and draw some conclusions.

\section{Definitions, assumptions and the general model}

The model, developed in Barnes and Roderick (2004), describes the dynamics of patch dry mass $Y$, where a patch is comprised of $n$ individual plants, each with dry mass $m_{j}(j=1 \ldots n)$, such that the dry mass of the entire patch is then $Y=n \bar{m}$. Individuals within each patch interact through a self-thinning mechanism, and the resulting model is given by

$$
\frac{\mathrm{d} Y}{\mathrm{dt}}=\bar{m} \frac{\mathrm{d} n}{\mathrm{dt}}+n \frac{\mathrm{d} \bar{m}}{\mathrm{dt}}=\left(\frac{1}{\bar{m}} \frac{\mathrm{d} \bar{m}}{\mathrm{dt}}\right)\left[\mathrm{Y}\left(1-\frac{\mathrm{Y}}{\kappa}\right)\right] .
$$

The existence of a patch carrying capacity $\kappa$ has been assumed, as well as a sigmoidal manner in which mass approaches this carrying capacity. (For full details of the formulation, see Barnes and Roderick, 2004, and for its application and validation see Barnes et al., in press.)

The model is of a logistic type, but with a nonlinear, timedependent 'reproduction rate' $(1 / \bar{m})(\mathrm{d} \bar{m} / \mathrm{dt})$, which emerges through the formulation of self-thinning. Further, we note that the coefficient $\mathrm{d} \bar{m} / \mathrm{dt}$, the rate of increase in the average mass of an individual, and the carrying capacity $\kappa$, incorporate response to available environmental resources. The steady state values for Eq. (1) occur at $\mathrm{Y}=0$ and $\kappa$, which (applying standard stability theory) are unstable and stable, respectively, for $\mathrm{d} \bar{m} / \mathrm{dt}>0$. Thus, if the system is disturbed from $\mathrm{Y}=\kappa$ it will return to this steady state over time. Note that, the per-capita growth rate, given by $(1 / \bar{m})(\mathrm{d} \bar{m} / \mathrm{dt})$, does not affect the steady state results; however, it does affect the rate at which the steady state is approached, in a nonlinear and time-dependent manner.

\section{Competing species and the intermediate disturbance hypothesis}

In response to clearing (or other disturbance) the successional recovery of a patch of vegetation typically involves changes in both the number of species and their abundance. Over time, certain species will dominate in terms of mass contribution, whilst other species, which may at first thrive, will largely die out. The model presented so far does not distinguish between species, but rather considers the total dry mass of all species combined. Below we show that the standard competing species model follows as a direct consequence of considering a number of species within the above formulation.

We examine first the case of two species, and then indicate how the developed theory can be extended to include many species.

\subsection{Theory for two species}

Using the dry mass formulation and notation of Section 2, let $Y=Y_{1}+Y_{2}$, where $Y_{s}=n_{s} \bar{m}_{s}(s=1,2)$ with $n_{s}$ the number of individuals, and $\bar{m}_{s}$ the average dry mass, of the sth species. Then, Eq. (1) becomes,

$$
\begin{aligned}
\frac{d Y}{d t} & =\frac{d\left(Y_{1}+Y_{2}\right)}{d t}=\left(\frac{1}{\bar{m}} \frac{d \bar{m}}{d t}\right)\left[Y\left(1-\frac{Y}{\kappa}\right)\right] \\
& =\left(\frac{1}{\bar{m}} \frac{d \bar{m}}{d t}\right)\left[\left(Y_{1}+Y_{2}\right)\left(1-\frac{\left(Y_{1}+Y_{2}\right)}{\kappa}\right)\right] \\
& =\left(\frac{1}{\bar{m}} \frac{d \bar{m}}{d t}\right)\left[Y_{1}\left(1-\frac{Y_{1}}{\kappa}\right)+Y_{2}\left(1-\frac{Y_{2}}{\kappa}\right)-2 \frac{Y_{1} Y_{2}}{\kappa}\right] .
\end{aligned}
$$

The last term of this expression describes the interaction between the species.

\subsubsection{Competing species}

We subdivide this equation into a system of two equations, describing the dynamics of $Y_{1}$ and $Y_{2}$, respectively:

$$
\begin{aligned}
& \frac{\mathrm{d} \mathrm{Y}_{1}}{\mathrm{dt}}=\left(\frac{1}{\bar{m}} \frac{\mathrm{d} \bar{m}}{\mathrm{dt}}\right)\left[\mathrm{Y}_{1}\left(1-\frac{\mathrm{Y}_{1}}{\kappa}\right)-\beta_{1} \frac{\mathrm{Y}_{1} \mathrm{Y}_{2}}{\kappa}\right], \\
& \frac{\mathrm{dY} \mathrm{Y}_{2}}{\mathrm{dt}}=\left(\frac{1}{\overline{\bar{m}}} \frac{\mathrm{d} \bar{m}}{\mathrm{dt}}\right)\left[\mathrm{Y}_{2}\left(1-\frac{\mathrm{Y}_{2}}{\kappa}\right)-\beta_{2} \frac{\mathrm{Y}_{1} \mathrm{Y}_{2}}{\kappa}\right],
\end{aligned}
$$

where $\beta_{1}+\beta_{2}=2$, and we will assume both parameters positive (see below). Equivalently,

$\frac{d Y_{1}}{d Y_{2}}=\frac{Y_{1}\left(\kappa-Y_{1}\right)-\beta_{1} Y_{2}}{Y_{2}\left(\kappa-Y_{2}\right)-\beta_{2} Y_{1}}$

This model takes the form of a competing species model (see, for example, Barnes and Fulford, 2002) with a time-dependent reproduction rate; the same for both species and equivalent to that for the whole community. Furthermore, the carrying capacity is common to both.

The terms involving $\beta_{1}$ and $\beta_{2}$ are the interaction terms, which, in this case, are dependent on the environmental 
conditions since $\mathrm{d} \bar{m} / \mathrm{dt}$ and $\kappa$ are part of their expression. Let

$\hat{\beta}_{\mathrm{S}}=\left(\frac{1}{\bar{m}} \frac{\mathrm{d} \bar{m}}{\mathrm{dt}}\right) \frac{\beta_{\mathrm{s}}}{\kappa}, \quad$ for $\quad s=1,2$.

Parameters $\hat{\beta}_{1}$ and $\hat{\beta}_{2}$ determine the manner in which one species influences the other. They can be interpreted as the rate at which one species renders resources unavailable to another (Barnes and Fulford, 2002). For example, the foliage of one species may reduce the light availability to others. To formulate this mathematically, define $\hat{\beta}_{1}$ as the rate at which species $Y_{2}$ blocks resources from species $Y_{1}$. Then the rate at which resources are made unavailable to species $Y_{1}$, by a single individual of $Y_{2}$, is given by $\hat{\beta}_{1} Y_{1}$, and by all of species $Y_{2}$ the rate is $\hat{\beta}_{1} Y_{1} Y_{2}$. Similarly, the rate at which $Y_{1}$ renders resources unavailable to $Y_{2}$ can be established as $\hat{\beta}_{2} Y_{1} Y_{2}$. This provides an interpretation for the interaction terms of Eq. (3) above, and it is recognized that there are many possible interpretations of resources for which the species may compete. We note that for $\mathrm{d} \bar{m} / \mathrm{dt} \approx 0$, there is no change in average mass. This coincides with no change in total mass $Y$ with time and subsequently no change in $Y$ due to competition (see Eq. (3)). The precise mechanisms, which are embodied by the interaction parameters, may be many and are varied; they will depend on the particular characteristics of the competitors and the nature of the disturbance (see, for example, Chesson, 2000b). However, the model presented above allows for this mechanistic variability, and could be extended to include other time-dependent or nonlinear phenomena.

The steady state values for the system of Eq. (3) provide a means of understanding how the species might dominate, coexist, or die out, over time. In the case $\beta_{1} \neq \beta_{2}$, the four steady states $\left(Y_{1}, Y_{2}\right)$ are:

$$
(0,0), \quad(0, \kappa), \quad(\kappa, 0) \text { and }\left(\frac{\kappa\left(\beta_{1}-1\right)}{\beta_{1} \beta_{2}-1}, \frac{\kappa\left(\beta_{2}-1\right)}{\beta_{1} \beta_{2}-1}\right) \text {. }
$$

Theoretically, the only possibility of stable coexistence occurs when $Y_{1}$ and $Y_{2}$ are both positive valued; that is, when $\beta_{1}=\beta_{2}=1$, whence $\mathrm{Y}_{2}=\kappa-\mathrm{Y}_{1}$ in the last steady state solution in (5). Alternatively, the two co-ordinates of this steady state are of opposite sign and thus not biologically relevant. (From standard stability theory the steady state at $(0,0)$ is an unstable node, while points $(\kappa, 0)$ and $(0, \kappa)$ are the end-points of the 'steady state line' $Y_{2}=\kappa-Y_{1}$.) Biologically, any positive initial conditions for the two species will result in coexistence. One species may dominate, but both will persist, with the steady state solution on the line $Y_{1}+Y_{2}=\kappa$, and each distinct pair of initial conditions resulting in a different steady state.

Alternatively, when $\beta_{1} \neq \beta_{2}$, one of the two species will dominate, while the other dies out. If $0<\beta_{1}<1$ (whence $1<$ $\left.\beta_{2}<2\right)$, then the system will approach a stable steady state at $(\kappa, 0)$; that is, species $\mathrm{Y}_{1}$ will dominate with time, and $\mathrm{Y}_{2}$ will die out. Applying standard stability theory, the steady state at $(0,0)$ is an unstable node, while that at $(0, \kappa)$ is a saddle point. Note that variation in $(1 / \bar{m})(\mathrm{d} \bar{m} / \mathrm{dt})$ will influence the rate at which the steady state is approached, but will not alter the structural dynamics.

As a consequence of this model, as illustrated by the dark lines in Fig. 1(a), depending on the initial numbers (mass) of the two species, the inferior species $\mathrm{Y}_{1}$ may dominate initially before dying out. This can be predicted as follows. Suppose that initially $\mathrm{Y}_{2} \ll \mathrm{Y}_{1} \ll \kappa$, then (initially), for $\mathrm{s}=1,2$,

$$
\begin{aligned}
& \frac{\mathrm{d} Y_{S}}{\mathrm{dt}} \approx\left(\frac{1}{\bar{m}} \frac{\mathrm{d} \bar{m}}{\mathrm{dt}}\right) Y_{S}>0 \\
& \Rightarrow Y_{S} \approx A_{S} \bar{m} \quad\left(A_{S}=\ln \left(\frac{Y_{S}(0)}{\bar{m}(0)}\right) \text { constant }\right) .
\end{aligned}
$$

Thus, $Y_{S}$ increases approximately linearly with $\bar{m}$ (assuming $(1 / \bar{m})(\mathrm{d} \bar{m} / \mathrm{dt})>0)$, with $\mathrm{Y}_{1}(0) \gg \mathrm{Y}_{2}(0)$ so that, initially, $\mathrm{Y}_{1}>\mathrm{Y}_{2}$. As both $Y_{1}$ and $Y_{2}$ increase, the above approximation is no longer valid, and then $d Y_{1} / d t$ becomes negative before $Y_{1} \rightarrow 0$. The relative growth rate $(1 / \bar{m})(\mathrm{d} \bar{m} / \mathrm{dt})$ was set constant for this figure, since (see Eq. (4)) it does not impact on the dynamical structure.

Fig. 1(b) illustrates the trajectory behaviour in the $\left(Y_{1}, Y_{2}\right)$ plane (the phase plane), for the case of model (3). Clearly $\mathrm{Y}_{2}$, the dominant species, continuously increases with time, while $\mathrm{Y}_{1}$ (the dominated species) increases initially, and then decreases to zero. What this figure contributes is that, using the dark arrows which indicate the direction of the trajectories, it can be seen that for any initial conditions within the plane, the system will approach $\left(Y_{1}, Y_{2}\right)=(0, \kappa)=(0,1)$ over time. Here the lower dashed line represents the locus of points such that $d Y_{1} / d t=0$, and similarly for the upper dashed line $\mathrm{dY}_{2} / \mathrm{dt}=0$.

It should be noted here that system (3) assumes that both species may attain the system carrying capacity of $\kappa$ in the absence of the other. In many systems, species cannot attain the same mass $\kappa$ in the absence of others; however, with $Y_{1}$ and $\mathrm{Y}_{2}$ representing the inferior and superior species, respectively, the system could equally have been given as,

$$
\begin{aligned}
\frac{d Y_{1}}{d t} & =\left(\frac{1}{\bar{m}} \frac{d \bar{m}}{d t}\right)\left[Y_{1}\left(1-\frac{Y_{1}}{\kappa_{1}}-\beta_{1} \frac{Y_{2}}{\kappa}\right)\right], \\
\frac{d Y_{2}}{d t} & =\left(\frac{1}{\bar{m}} \frac{d \bar{m}}{d t}\right)\left[Y_{2}\left(1-\frac{Y_{2}}{\kappa}-\beta_{2} \frac{Y_{1}}{\kappa}\right)+\frac{Y_{1}^{2}}{\kappa_{1}}-\frac{Y_{1}^{2}}{\kappa}\right] \\
& =\left(\frac{1}{\bar{m}} \frac{d \bar{m}}{d t}\right)\left[Y_{2}\left(1-\frac{Y_{2}}{\kappa}-\beta_{2} \frac{Y_{1}}{\kappa}\right)+\frac{Y_{1}^{2}\left(\kappa-\kappa_{1}\right)}{\kappa \kappa_{1}}\right],
\end{aligned}
$$

where $0<\kappa_{1}<\kappa$ is the carrying capacity for the inferior species $Y_{1}$. The numerical results are illustrated in Fig. 1(a) by the fine lines. Note that the impact of including this lower carrying capacity for the inferior species, with all other conditions unchanged, results in the dominated species peaking earlier with a lower maximum, and the dominant species approaching its carrying capacity more rapidly. This approach can be extended to include reduced carrying capacities for both species; both below $\kappa$ pertaining to the whole community.

\subsubsection{The intermediate disturbance hypothesis}

At this point we can conjecture that, maintaining the system in the left hand segment of Fig. 1(a), would provide the conditions for the constant regeneration of less dominant species, and the results would be consistent with the intermediate disturbance hypothesis at a patch scale. This assumes that disturbance alters the competitive balance between species, such that the inferior competitors gain a relative advantage, thus preventing competitive exclusion over the long term. 

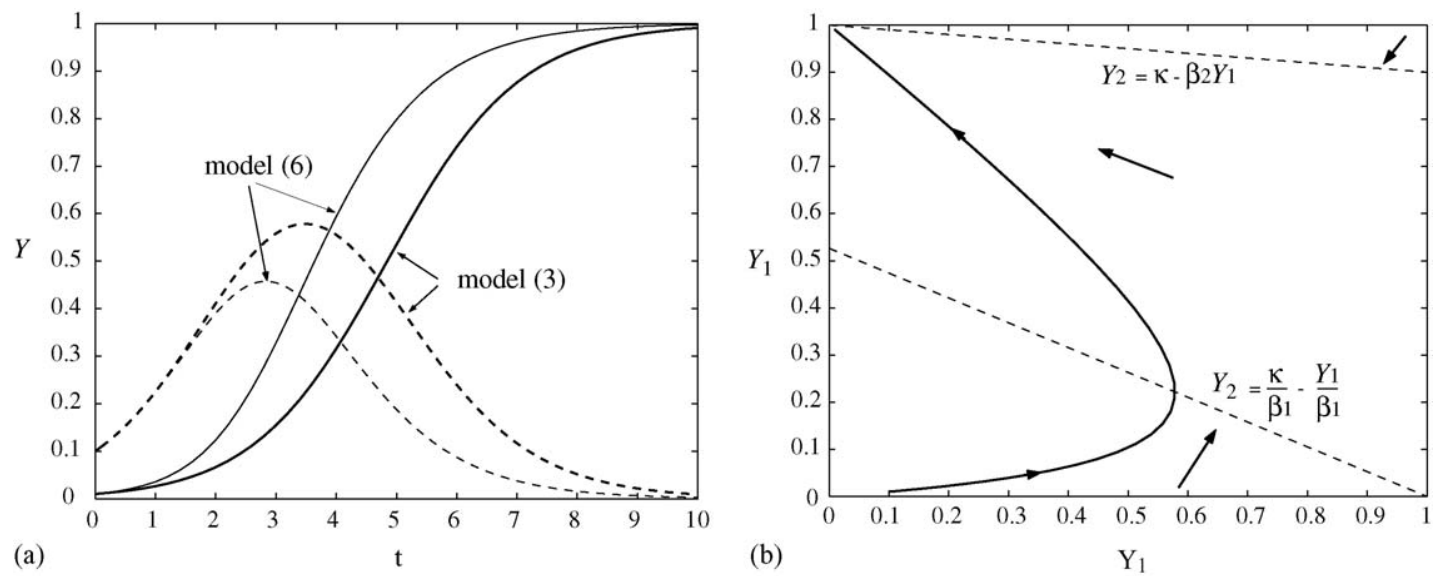

Fig. 1 - (a) Application of models (3) (dark lines) and (6) (fine lines) illustrate that an inferior species ( $Y_{1}$, dashed lines) may dominate initially, but the superior species $\left(Y_{2}\right.$, solid lines) will dominate over time. (b) Plot of the $\left(Y_{1}, Y_{2}\right)$ phase plane for model (3), with the dark line tracing out the trajectory over time. The arrows indicate the direction of trajectories in that region of the plane, and the dashed curves are the lines used to establish the fourth steady state. For both (a and $b$ ), the parameter values are $\beta_{1}=1.9, \beta_{2}=0.1, \kappa=1, \kappa_{1}=0.8$, with initial conditions $\left(\mathrm{Y}_{1}, \mathrm{Y}_{2}\right)=(0.1,0.01)$ and $(1 / \overline{\mathrm{m}})(\mathrm{d} \overline{\mathrm{m}} / \mathrm{dt})=1$ assumed constant.

To gain some understanding of the impact of frequent disturbance, we include regular disturbance into model (3); that is, the removal of mass from the system intermittently. Disturbance of this type can be considered at any time scale, from daily to time periods of centuries:

$$
\begin{aligned}
& \frac{d Y_{1}}{d t}=\left(\frac{1}{\bar{m}} \frac{d \bar{m}}{d t}\right)\left[Y_{1}\left(1-\frac{Y_{1}}{\kappa}-\beta_{1} \frac{Y_{2}}{\kappa}\right)\right]-\gamma_{1} Y_{1}, \\
& \frac{d Y_{2}}{d t}=\left(\frac{1}{\bar{m}} \frac{d \bar{m}}{d t}\right)\left[Y_{2}\left(1-\frac{Y_{2}}{\kappa}-\beta_{2} \frac{Y_{1}}{\kappa}\right)\right]-\gamma_{2} Y_{2},
\end{aligned}
$$

where $0<\gamma_{i}<1$, with $i=1$, 2 . Such a disturbance may describe the removal of vegetation mass from the system through events such as fire, herbivory, disease or cropping, in which case a proportion $\left(\gamma_{1}\right.$ or $\gamma_{2}$ ) of the current mass is removed. Since any disturbance impacts on different species to varying degrees (for example, herbivory may effect only a particular species, fire or clearing may have a greater effect on the smaller or larger species, respectively, with the seedbanks retained), the rates are taken as different for the different species. This provides a phenomenological description of the outcome of any one of several potential underlying fluctuation dependent mechanisms of species coexistence (Shea et al., 2004). Typical results are illustrated in Fig. 2(dark lines) where, for the chosen parameters, the system stabilizes with both species coexisting.

We have shown that the only possible stable coexistence steady state for model (3) occurred for $\beta_{1}=\beta_{2}=1$. We now show that, when disturbance is included, a stable state of coexistence can be predicted for $\beta_{1} \neq \beta_{2}$, where we assume, without loss of generality, that $0<\beta_{2}<1<\beta_{1}<2$. Further, we establish that diversity (coexistence) does not occur at very low or high intensities of disturbance, but only for an intermediate range.

As for model (3), we can establish four steady state points for the system, only one of which describes possible coexis- tence and is given by:

$\left(Y_{1}, Y_{2}\right)=\left(\frac{-\kappa\left(m-m \beta_{1}+\beta_{1} \gamma_{2}-\gamma_{1}\right)}{m\left(\beta_{1} \beta_{2}-1\right)}, \frac{-\kappa\left(m-m \beta_{2}+\gamma_{1} \beta_{2}-\gamma_{2}\right)}{m\left(\beta_{1} \beta_{2}-1\right)}\right)$,

where $m=(1 / \bar{m})(\mathrm{d} \bar{m} / \mathrm{dt})$. Note that, if $\beta_{1} \neq \beta_{2}$, then $\beta_{1} \beta_{2}-1=$ $\beta_{1}\left(2-\beta_{1}\right)-1=-\left(\beta_{1}-1\right)^{2}<0$, so that both coordinates are positive (and thus biologically relevant), if

$\beta_{1}\left\{\begin{array}{lll}<\frac{m-\gamma_{1}}{m-\gamma_{2}}, & \text { if } & m>\gamma_{2} \\ >\frac{m-\gamma_{1}}{m-\gamma_{2}}, & \text { if } & m<\gamma_{2}\end{array}\right.$
$\beta_{2}\left\{\begin{array}{lll}<\frac{m-\gamma_{2}}{m-\gamma_{1}}, & \text { if } & m>\gamma_{1} \\ >\frac{m-\gamma_{2}}{m-\gamma_{1}}, & \text { if } & m<\gamma_{1}\end{array}\right.$

Note that $\beta_{1}=2-\beta_{2}, 0<\beta_{1}<2$ and $0<\gamma_{1,2}<1$.

If the trace of the Jacobian matrix associated with the system, evaluated at the steady state (8), is negative, then that point is stable. The trace of this matrix evaluated at steady state (8) simplifies to,

$\operatorname{trace}(J)=\frac{\gamma_{2}-\gamma_{1}}{\beta_{2}-1}$

Thus, the point is stable when $\left(\gamma_{2}-\gamma_{1}\right)$ and $\left(\beta_{2}-1\right)$ are of opposite sign, rendering the trace negative. Without loss of generality (as above) $0<\beta_{2}<1<\beta_{1}<2$; thus, coexistence requires $\gamma_{2}>\gamma_{1}$.

The dark lines in Fig. 2(a), with parameters as given in the caption, provide one example of the system where a stable steady state is attained in which both species coexist. (Applying standard stability theory for this parameter combination it can be established that the steady state at the origin 

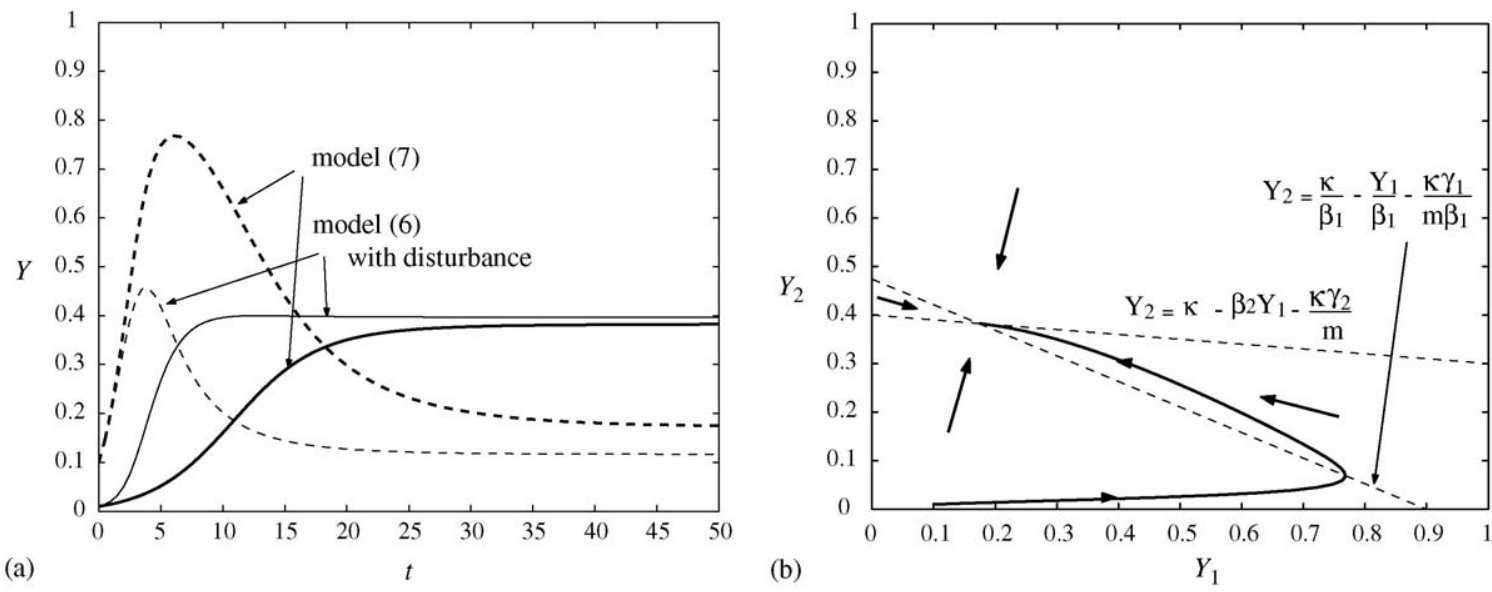

Fig. 2 - (a) Model (7) (which includes disturbance) is illustrated with dark lines, and model (6) including a lowered carrying capacity $\kappa_{1}$ and disturbance, with fine lines. Solid lines refer to the superior species and dashed lines the inferior species. (b) Plot of the $\left(Y_{1}, Y_{2}\right)$ phase plane for model (7), with the dark line tracing out the trajectory over time. The arrows indicate the direction of trajectories in that region of the plane, and the dashed curves are the lines used to establish the fourth steady state. Parameter values for ( $a$ and $b$ ) are as for Fig. 1 with $\gamma_{1}=0.1$ and $\gamma_{2}=0.6$.

is unstable, while the two steady states for which only one species survives are unstable saddle points.) The fine lines represent the case where the less dominant species has a lowered carrying capacity $\kappa_{1}$ (model (6) with disturbance), and is provided for the purposes of comparison, although the analytic details are not included. In Fig. 2(b) we plot the $\left(\mathrm{Y}_{1}, \mathrm{Y}_{2}\right)$ plane for system (7). As previously, the arrows indicate the direction of the trajectories in the plane, and it can be seen that for all initial conditions in the plane the trajectories will approach the same steady state (located at the intersection of the dashed lines).

While Fig. 2( $a$ and $b$ ) illustrate results for a particular set of parameters, and constant $m$, it is of interest to establish for variable $m$ the interval of the disturbance parameter for which coexistence occurs. Since $\mathrm{d} \bar{m} / \mathrm{dt}$ (without disturbance) approaches zero over time, and since disturbance impacts on both $\bar{m}$ and $d \bar{m} / d t$, we have adopted a sinusoidal function of time to simulate the dynamics of this term:

$\frac{1}{\bar{m}} \frac{d \bar{m}}{d t} \approx \frac{1}{2}(1+\sin (t))$.

We note that $\sin (t)$ does not have the same period as the system disturbance. If it did, this would be equivalent to the inclusion of a constant $(1 / \bar{m})(\mathrm{d} \bar{m} / \mathrm{d} t)$, and our purpose is to retain time dependence. To illustrate the model's agreement with the intermediate disturbance hypothesis, we vary the disturbance intensity on the superior competitor $\left(\gamma_{2}\right)$, and then relate this to an index of community diversity $D$ (Simpson's $D$; Begon et al., 1986). With parameter values as in the caption, our results indicate that maximum diversity peaks at an intermediate level of $\gamma_{2}$ (Fig. 3).

\subsubsection{Comparison with other modelling approaches}

The significance of the results above derives in part from the generality of this modelling approach. Shea et al. (2004) reviewed the literature for evidence in support of the intermedi- ate disturbance hypothesis, classifying studies as either observational, experimental, or theoretical. In their review, the studies identified as theoretical all shared a common trait, whereby coexistence-promoting differences among the species in their response to disturbance were explicitly represented within the model equations. For example, Caswell and Etter (1999) and Roxburgh et al. (2004) both used a cellular automata approach, with species differing in their spatial dispersal and competitive abilities. More recently, Yamamoto and Hatta (2004) modelled phytoplankton diversity in response to temporal fluctuations in nutrient availability, with the model parameters defining differences in the species affinities to and ability to process different nutrients. One of the main aims of these studies is therefore to gain insight into how particular differences in the species' ecologies can contribute to coexistence,

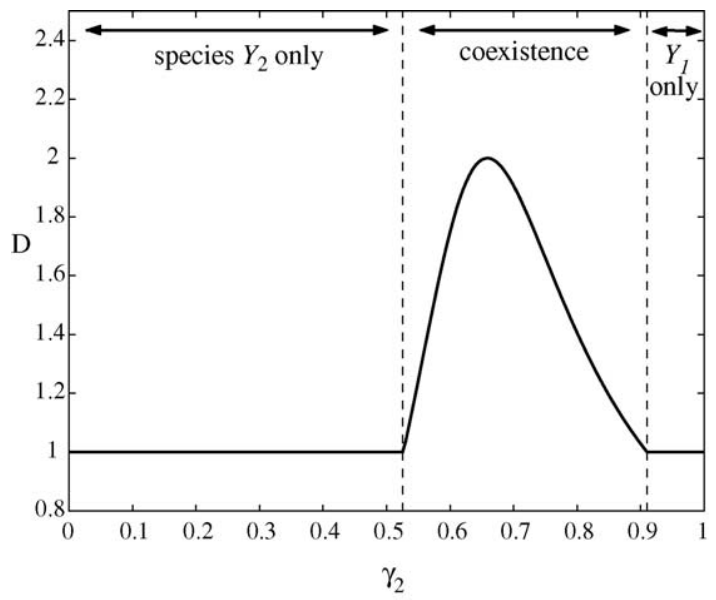

Fig. 3 - With the Simpson diversity index $D$, model (7), including disturbance and variable $(1 / \bar{m})(\mathrm{d} \bar{m} / \mathrm{dt})$, predicts an intermediate level of disturbance intensity $\gamma_{2}$ for which $D$ is maximized. Other parameters were set as for Fig. 1, with $\gamma_{1}=0.1$. 
thus leading to a greater mechanistic understanding of the process.

This contrasts with the model presented here, where we seek to explore, in the most general way possible, the impacts of disturbance on the model dynamics. We note that, regardless of the specific ecological differences underlying the coexistence, the net consequence is that disturbance allows the expression of spatial and/or temporal niches (Roxburgh et al., 2004), resulting in a decline in the strength of between-species competition relative to within-species competition (Chesson, $2000 \mathrm{~b}$ ). In the model presented here, these consequences are modelled directly as species-specific susceptibilities to disturbance (Eq. (7), $\gamma_{1}$ and $\gamma_{2}$ ). Such explicit favouring of the weaker competitors provides the opportunity for coexistence, but does not pre-determine the unimodal disturbance-diversity pattern characteristic of the intermediate disturbance hypothesis, which, as noted earlier, emerges from the model formulation. The main advantage of our approach lies in its generality; the price of this generality is, however, that much of the underlying ecology remains hidden within the parameters $\gamma_{1}$ and $\gamma_{2}$.

\subsection{Theory for $\mathrm{N}$ species}

We return to the theory described at the beginning of the previous section, Eq. (3), and extend it to include $N$ species. The general model, for the sth species, becomes

$$
\frac{\mathrm{d} Y_{\mathrm{S}}}{\mathrm{dt}}=\left(\frac{1}{\bar{m}} \frac{\mathrm{d} \bar{m}}{\mathrm{dt}}\right)\left[\mathrm{Y}_{\mathrm{S}}\left(1-\frac{\mathrm{Y}_{\mathrm{S}}}{\kappa}-\frac{1}{\kappa} \sum_{\substack{r=1 \\ r \neq s}}^{N} \beta_{\mathrm{sr}} Y_{\mathrm{r}}\right)\right],
$$

with $\beta_{\text {sr }}+\beta_{r s}=2$.

As above, there is only one steady state for which all species coexist. This point is not biologically feasible if there is some $\beta_{\text {sr }} \neq 1$, since then one of the coordinates is negative. Alternatively, when all $\beta_{S r}=1$ then the steady state is given by the surface $0=1-\sum_{S} Y_{S} / \kappa$, which can be established as stable, and for each set of initial conditions there is a different steady state with all species persisting. Further steady states have $Y_{s}=0$ for some $s$, and then the analysis follows from that of $N-1$ species. That is, all species with interaction parameters $\beta_{\text {sr }}=1=\beta_{\text {rs }}$ will coexist whilst the others will die out over time. Thus from the case of two species discussed earlier, and induction, we can establish the behavior for $\mathrm{N}$ species.

As with the case of two species, the only possible steady state with all species coexisting is attained when all interaction parameters are equal, and the mass contribution from each species will depend on the initial conditions, that is, the available seed. This scenario, for the case of three species, is illustrated in Fig. 4(a). Alternatively, certain species may survive whilst others die out. One example is given in Fig. 4(b), where, for the case of three species, one eventually dominates over the other two. It should be noted that the other two species flourish before dying out, and again there is an intermediate stage where all three species are abundant.

\subsection{Discussion and conclusions}

Splitting the dynamical equation for $Y$ (total dry mass in a vegetation patch) into contributions from different species, we have shown that the competing species model emerges, and that the steady state solutions predict where certain species may coexist, or where superior species may dominate, whilst others die out. This process is illustrated in Figs. 1 and 4, for two and three species, respectively. In general, this model formulation for vegetation regeneration predicts a period where many species may flourish, followed by, over a long period of time, the domination of a few.

Disturbance and senescence provide the opportunity for the constant regeneration of the inferior competitor: in this sense disturbance may reduce competitive inequality, although this inequality may eventually dominate over long time periods. This implies that, whilst the model may predict extinction of an inferior species over time, fluctuations at smaller time scales, or disturbance, will ensure that it persists through regular regeneration. We showed that, in
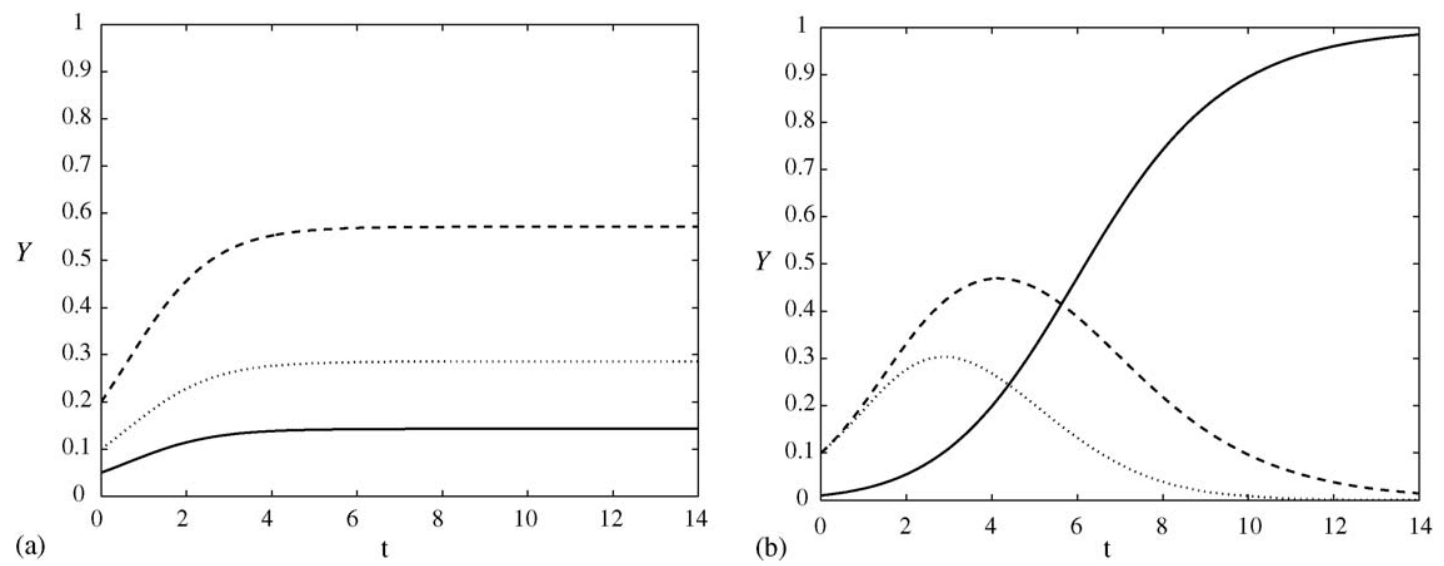

Fig. 4 - Application of model (9) illustrates the interaction of three species in vegetation regeneration: (a) where all species coexist over time $\left(Y_{1}\right.$, dashed line; $Y_{2}$, solid line; $Y_{3}$, dotted line), and with initial conditions $\left(Y_{1}, Y_{2}, Y_{3}\right)=(0.2,0.05,0.1)$ and parameter values $\beta_{12}=\beta_{13}=\beta_{23}=1$; (b) where there is an intermediary stage with an abundance of all species, followed by the domination by one, $Y_{2}$ (solid line) $\left(Y_{1}\right.$, dashed line; $Y_{3}$, dotted line), with initial conditions $\left(Y_{1}, Y_{2}, Y_{3}\right)=(0.1,0.01,0.1)$, and parameters $\beta_{12}=1.5, \beta_{13}=0.8, \beta_{23}=0.1$. For both (a and $\left.b\right), \kappa=1$, and $1 / \bar{m}(\mathrm{~d} \bar{m} / \mathrm{dt}$ ) $=1$ assumed constant. 
general, the model predicts that neither extremely high, nor low, disturbance intensities result in diversity; rather there is an intermediate disturbance parameter region, determined by the interaction parameter $\beta_{1}$ and relative growth rate $m$, for which diversity ensues. In a general sense, instabilities (that is, frequent disturbance) at shorter time scales may ensure the long-term stability (that is, persistence) of species diversity (Chesson and Huntly, 1989). While these results do not explain the mechanisms, they are in accordance with the predictions of the intermediate disturbance hypothesis for a vegetation patch, where disturbance here is temporal in nature. Figs. 2 and 3 illustrate this concept in the case of two species.

In summary, we have shown that by formulating the model in the way that is presented in this paper, self-thinning allows a natural scaling between the individual and group of individuals, or patch. The standard competing species model is a natural consequence of the mathematics of the approach, with the per-capita growth rate emerging explicitly in the governing equations in a nonlinear and time-dependent form. The ensuing mathematical predictions, concerning frequent disturbance and competitive inequality, are in agreement with the intermediate disturbance hypothesis.

\section{REFERENCES}

Barnes, B., Bi, H., Roderick, M. Application of an ecological framework linking scales across space and time based on self-thinning. Ecol. Appl., in press.

Barnes, B., Fulford, G., 2002. Mathematical Modelling with Case Studies. Taylor and Francis, London.
Barnes, B., Roderick, M., 2004. An ecological framework linking scales across space and time based on self-thinning. Theor. Population Biol. 66, 113-118.

Begon, M., Harper, J.L., Townsend, C.R., 1986. Ecology: Individuals, Populations and Communities. Black Scientific Publications, Boston.

Caswell, H., Etter, R., 1999. Cellular automaton models for competition in patchy environments: facilitation, inhibition and tolerance. Bull. Math. Biol. 61, 625-649.

Chesson, P., 1994. Multispecies competition in variable environments. Theor. Population Biol. 45, 227-276.

Chesson, P., 2000. General theory of competitive coexistence in spatially-varying environments. Theor. Population Biol. 58, 211-237.

Chesson, P., 2000. Mechanisms of maintenance of species diversity. Annu. Rev. Ecol. Syst. 31, 343-366.

Chesson, P., Huntly, N., 1989. Short-term instabilities and long-term community dynamics. Trends Ecol. Evol. 4 (10), 293-298.

Clark, J.S., 1993. Shifting mosaic metapopulation dynamics. In: Levin, S., Powell, T., Steele, J. (Eds.), Patch Dynamics. Springer-Verlag, Berlin, pp. 224-246.

Niklas, K., 1994. Plant Allometry: The Scaling of Form and Process. University of Chicago Press, Chicago.

Niklas, K., Enquist, B., 2002. Canonical rules for plant organ biomass partitioning and annual allocation. Am. J. Bot. 89 (5), 812-819.

Roxburgh, S.H., Shea, K., Wilson, J.B., 2004. The intermediate disturbance hypothesis, patch dynamics and mechanisms of species coexistence. Ecology 85, 359-371.

Shea, K., Roxburgh, S.H., Rauschert, E.S.J., 2004. Moving from pattern to process: coexistence mechanisms under intermediate disturbance regimes. Ecol. Lett. 7, 491-508.

Yamamoto, T., Hatta, G., 2004. Pulsed nutrient supply as a factor inducing phytoplankton diversity. Ecol. Modell. 171, 247-270. 